\title{
Das EPD und die Datenflut
}

\section{Yvonne Gilli}

Dr. med., Mitglied des FMH-Zentralvorstandes, Departement Digitalisierung / eHealth

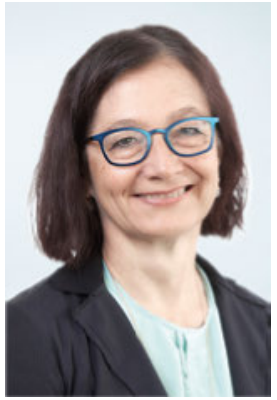

Der Psychologe Stanley Milgram schrieb Geschichte mit seinem provozierenden Experiment zu Gehorsam gegenüber Autoritäten, in dem Versuchspersonen Mitmenschen auf Aufforderung mit Elektroschocks bestraften. Die Wissenschaftscommunity reagierte kontrovers auf sein traumatisierendes Experiment, das aus heutiger Sicht einzig im historischen Kontext der Aufarbeitung der Nazi-Verbrechen überhaupt verständlich ist. Milgram selbst distanzierte sich später von dieser sozialpsychologischen Forschungsarbeit. Weniger bekannt sind spätere Arbeiten von ihm, unter anderem formulierte er bereits in den 70er Jahren die «Urban-Overload-Hypothesis», wonach Menschen in Städten wegen Reizüberflutung ihr soziales Verhalten änderten. Vielleicht ist es Zeit, diese Hypothese neu zu reflektieren in einer Zeit des Digital-Information-Overload. Sieht man davon ab, wie viel Gesundheitsdaten

Spitäler werden wesentlich mehr Daten im EPD verfügbar machen, als der klassische Austrittsbericht umfasst.

durch Wearables erzeugt werden, so bewegen wir uns zwischenzeitlich in einzelnen Spitälern im ein- bis zweistelligen Petabyte-Bereich digitaler Daten.

Eine Studie des Veterans Affairs Medical Center aus dem Jahr 2010 hat einen Zusammenhang zwischen dem Übersehen von Meldungen und einem ,Information Overload' in Electronic Health Records konkludiert. Mit dem elektronischen Patientendossier (EPD) wird der Ärzteschaft eine neue, zusätzliche digitale Informationsquelle zur Verfügung stehen. Schon jetzt zeichnet sich ab, dass Spitäler mehr Daten im EPD der Patientinnen und Patienten verfügbar machen werden, als der klassische Austrittsbericht umfasst. Diese Entwicklung ist Ausdruck eines digitalen Wandels, bei dem die digitalisierten Befunde oder auch Einzelwerte rasch aggregiert, kumuliert und transportiert werden können.

Der amerikanische Publizist Clay Shirky hat die Lösung für das Problem der zunehmenden Informationsflut auf einen Nenner gebracht: "It's not information overload. It's filter failure.» Dieses Paradigma funktioniert in der Welt von Social Media, und auch ein Spamfilter in meinem Postfach verrichtet mir nützliche Arbeit. Kaum vorstellbar, jedenfalls nicht in naher Zukunft, ist dies für das EPD, um die zunehmende Anzahl und die Komplexität der behandlungsrelevanten Daten über die Zeit zu reduzieren.

Weder äussert sich das EPDG und die erlassenen Verordnungen konkret darüber, welche Daten im EPD zu erfassen sind, noch nehmen sie Stellung zur Frage, wie weit die Ärzteschaft verpflichtet ist, das EPD im Rahmen ihrer Behandlungstätigkeit als Informationsquelle zu nutzen. Der gewollte Verzicht auf eine Konkretisierung stellt die am EPD teilnehmenden Ärzte vor die Herausforderungen, die Funktion des EPD zu erfüllen und gleichzeitig den datenschutzrechtlichen Vorgaben sowie dem Arztgeheimnis, unter Androhung der im StGB aufgeführten Strafen, Rechnung zu tragen. Dies alles ist eine Frage der beruflichen Sorgfaltspflicht, die aus nachvollziehbaren Gründen nicht im EPDG geregelt ist, jedoch im Einzelfall beurteilt werden muss. Da im EPD jede Erfassung und Veränderung von Daten aufgezeichnet werden, ist ein Dokumentationsfehler im Zusammenhang mit dem EPD einfach nachzuweisen.

Das Fehlen konkreter Vorgaben im Umgang mit dem EPD bedeutet für die Ärztinnen und Ärzte, die sich freiwillig der EPD-Infrastruktur anschliessen, ein derzeit in seiner Tragweite noch unklares Haftungsrisiko.

\section{Rechtliche Unsicherheiten könnten einen} Teil der Ärzteschaft veranlassen, von einem Anschluss an die EPD-Infrastruktur abzusehen.

Diese rechtlichen Unsicherheiten könnten einen erheblichen Teil der Ärzteschaft dazu veranlassen, entweder von einem Anschluss an die EPD-Infrastruktur abzusehen, oder umgekehrt, alle relevanten Daten ins EPD zu stellen, zumal die zukünftige Relevanz der Daten zum Zeitpunkt der Datenerfassung nicht mit letzter Gewissheit prognostiziert werden kann. Beide Vorgehensweisen begründen sich mit Haftungsrisiken, behindern aber eine nutzenbringende Kommunikation zwischen Gesundheitsfachpersonen. 